\title{
HIGH-SPEED, LARGE SCAN AREA, DISTORTION-FREE OPERATION OF SINGLE- CHIP ATOMIC FORCE MICROSCOPES
}

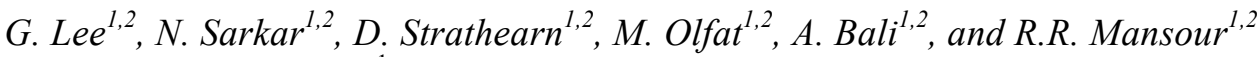 \\ ${ }^{1}$ University of Waterloo, Canada \\ ${ }^{2}$ ICSPI Corp., Canada
}

\begin{abstract}
We report a 50 -fold increase in the scan speed and a $200 \%$ improvement in the distortion-free scan range of a single-chip atomic force microscope (sc-AFM). The thermal time constants of 3 -DOF actuators have been reduced by a factor of $\sim 10$ to increase the bandwidth of quasi-static AFM modes, while also increasing efficiency in dynamic modes. Importantly, the lifetime of the instrument is shown to exceed that of a conventional AFM cantilever by a factor of $>100 x$. These devices have produced the first sc-AFM images of a $22 \mathrm{~nm}$ SRAM bank on an Intel Ivy Bridge CPU.
\end{abstract}

\section{INTRODUCTION}

Despite the exquisite resolution that may be obtained with AFMs, the industrial nanometrology enterprise has been reluctant to include them in their suite of inspection tools. The single-chip AFM (sc-AFM) [1] was introduced to overcome several shortcomings of conventional AFMs by replacing bulky piezoelectric scanners with 3 degree-of-freedom electrothermal (ET) MEMS actuators and by replacing laser detection with thermal-piezoresistive resonant sensing. These single-chip instruments are well-suited to high-speed operation and may also be implemented as arrays.

Perhaps the most significant drawback of conventional AFMs, when compared to other scanning microscopes such as confocal or scanning electron microscopes, is that it takes 1-100 minutes to obtain a high-quality AFM image. The first paper on high-speed AFM was published 25 years ago [2]. Several highly customized scanners were then developed to demonstrate video-rate AFM $[3,4]$. These instruments place severe constraints on the sample volume because the reported scanners cannot accommodate large masses (i.e. wafers). The present work obviates any sample movement because the tip is scanned in 3DOF by MEMS actuators, thus markedly raising the lowest natural frequency of the instrument.

Tip wear is an important concern in AFM productivity. Along with the increased cost of consumables, the tip-exchange process is both time-consuming and tedious. The user must first install a small silicon die into a custom fixture, and then align a laser to reflect from the surface of a small cantilever onto a photodiode. The reported device may be used for over 9000 scans of a sample with no visible tip wear in intermittent contact mode. Furthermore, the tip exchange process does not require any finesse, as the force sensor is integrated on-chip.

\section{DEVICE GEOMETRY}

The geometry of the reported instrument is shown in Fig.1. Lateral actuators are arranged in isothermal pairs to enable scanning at a constant temperature [5], thereby suppressing thermal coupling to piezoresistive sensors. A lever arrangement provides sufficient geometric advantage to obtain $15 \times 15 \mu \mathrm{m}$ scans. A range of $>60 \mu \mathrm{m}$ may be obtained as a single isothermal line profile, and scanning with various aspect ratios has been demonstrated.

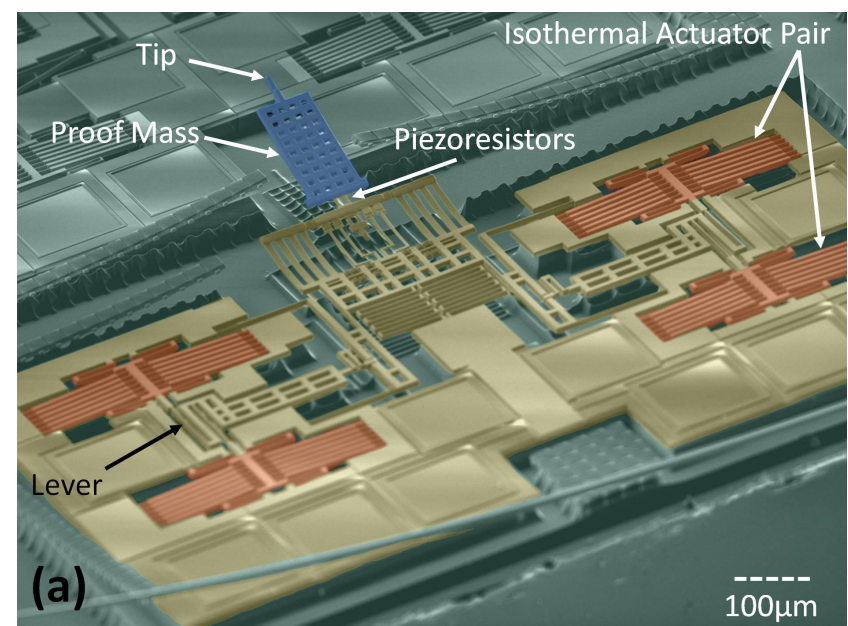

Figure 1: False color SEM image of a single-chip AFM with several components highlighted.

A stiffer flexural suspension yields a first lateral mode that is $\sim 2 \mathrm{x}$ higher than the cantilever's natural frequency (Fig.2); the opposite is true for AFMs reported to date, which suffer from deleterious resonances at high scan speeds. a)

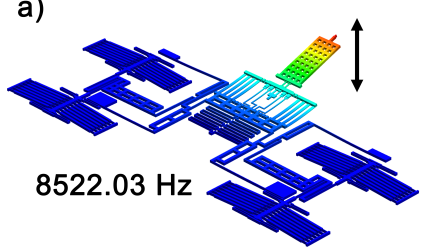

b)

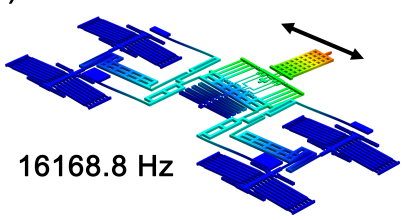

Figure 2a) First mode of the Sc-AFM is a vertical resonance and b) second mode is a lateral resonance.

Optimal placement of heaters within actuators reduces their thermal time constants by an order of magnitude to enable fast scanning (Fig.3). In previous design iterations, the polysilicon heaters in the chevron were located within the shuttle region. Current renditions of the design place the heaters within contoured beams of the chevron. The temperature distribution of the chevron allows for $3 \mathrm{x}$ the amount of displacement with similar force output before mechanical failure occurs when thermal budgets are exceeded. Also, while the heat capacity of both chevrons is similar, the thermal resistance from the heater to the active material is much lower in the present design, increasing the bandwidth of the actuator. This allows the actuator to operate at higher frequencies without compromising the scan range. 


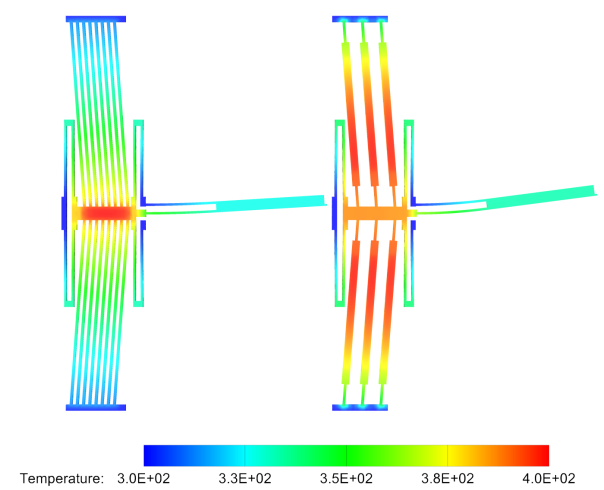

Figure 3: Temperature distribution within different chevron actuators. The left actuator shows a heater located within the shuttle of the device whereas the right actuator shows the heaters located within the contoured chevrons.

Similarly, the heater resistor location in the Z-actuator has been optimized such that sensor data can be captured at faster rates. Placing the heater beams directly under a vertical bimorph can help to distribute the heat, allowing for a faster response time (Fig.4).

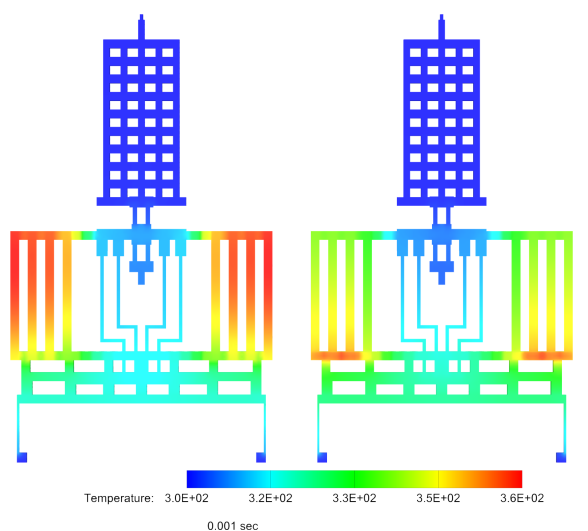

Figure 4: Transient heat distribution after $1 \mathrm{~ms}$ of similar heat flux. The actuator on the left contains heaters that are located directly under the vertical bimorph whereas the right device contains heaters that are located at the base of the vertical bimorph. The quicker temperature generation over the bimorph allows for a faster reaction speed.

\section{IMAGING RESULTS}

Buried vias of a $0.35 \mu \mathrm{m}$ CMOS process were imaged using the sc-AFM to demonstrate a scan range of roughly $15 \times 15 \mu \mathrm{m}$. Each of the $0.5 \mu \mathrm{m}$ vias are spaced at a pitch of $1 \mu \mathrm{m}$ (Fig.5a). The topography of an air-bearing surface on a hard-disk reader head was imaged (Fig. 5b). The varying rates of material removal in the lapping process result in the observed patterns. Atomic lattice steps on $\mathrm{SiC}$ with $1.5 \mathrm{~nm}$ height have also been captured to demonstrate the vertical sensitivity of the sc-AFM (Fig.5c). The sc-AFM has also imaged various biological samples including the compound eye of a butterfly, where small striations within the ommatidium serve as an antireflective coating (Fig.5d).
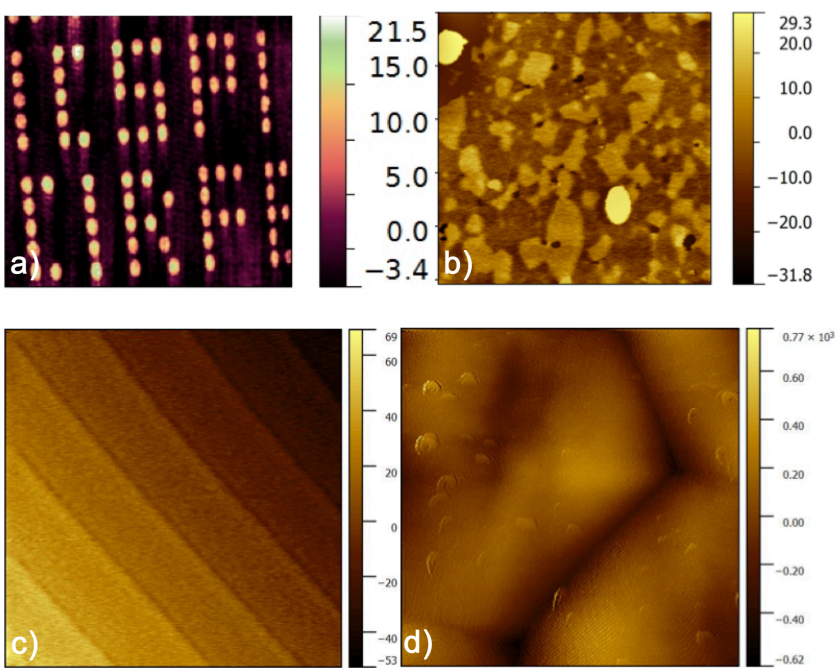

Figure 5a) Buried vias spelling ICSPI and CIRFE, b) Hard drive read head, c) $1.5 \mathrm{~nm}$ tall SiC lattice steps, d) Compound eye of butterfly

\section{QUASI-STATIC OPERATION}

Vertical actuators with short thermal time constants have enabled the acquisition of tip-sample interaction dynamics at high data rates for the first time with an sc-AFM (Fig.6). Once the tip and sample are engaged, compressive stress in the cantilever may be measured to reveal topography. Upon retraction, the abrupt transition from attractive tip-sample interaction forces to freeoscillation is captured. The harder the contact, the more the cantilever must retract before the tip detaches from the sample. This information may be used to reconstruct images of the topography, adhesion, and dissipation in the sample simultaneously in a single pass.

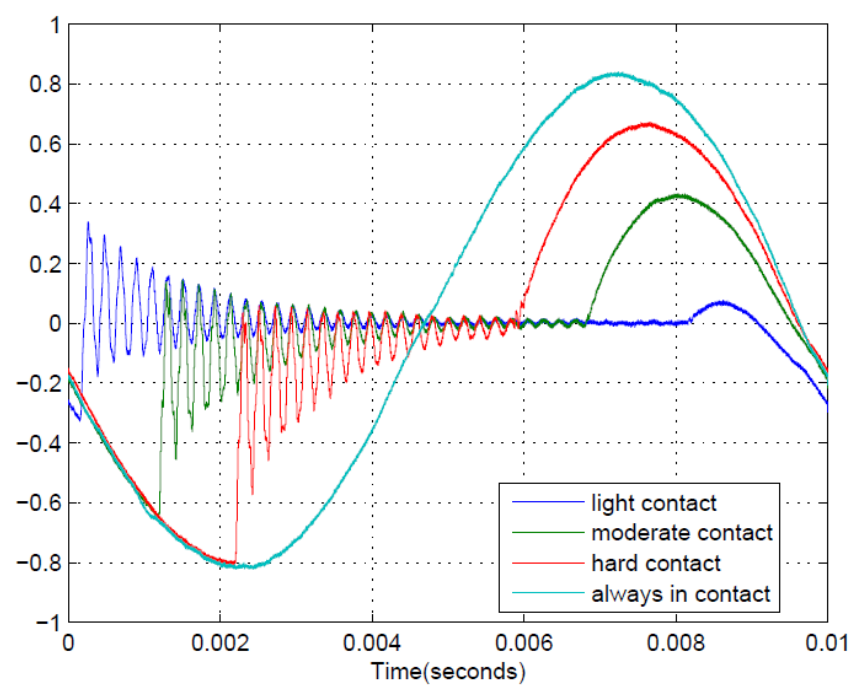

Figure 6: The free-oscillation of the sc-AFM cantilever is captured with the integrated polysilicon resistors after abruptly detaching from a surface with varying degrees of contact force. 


\section{HIGH-SPEED OPERATION}

To date, high-speed AFM research has employed contact mode, which causes rapid tip wear. The sc-AFM may be operated at higher speeds than conventional instruments in intermittent contact mode (AM-AFM) as shown in Fig 7a. A square-rooted sinusoidal wave is used to drive the $\mathrm{Z}$-actuator into resonance in order to obtain an image that is enhanced by the $\mathrm{Q}$ factor of the device. The strain experienced in the piezoresistive bridge of the sc-AFM is used to adjust the PID such that the tip will be held at a constant height above the sample. Fig $7 \mathrm{~b}$ shows the line scan of a $100 \mathrm{~nm}$ calibration grating, obtained with progressively higher scan rates, to demonstrate that a $50 \mathrm{x}$ improvement in scan speed has been achieved. Beyond $30 \mathrm{~Hz}(60$ lines/sec), features appear rounded because of the low-pass filter at the output stage of the demodulator. The lateral range of data also diminishes overtime, as this device contains chevron actuators that do not have the optimized heater placement. The higher thermal resistance reduces the chevron bandwidth at higher frequencies of operation.

a)

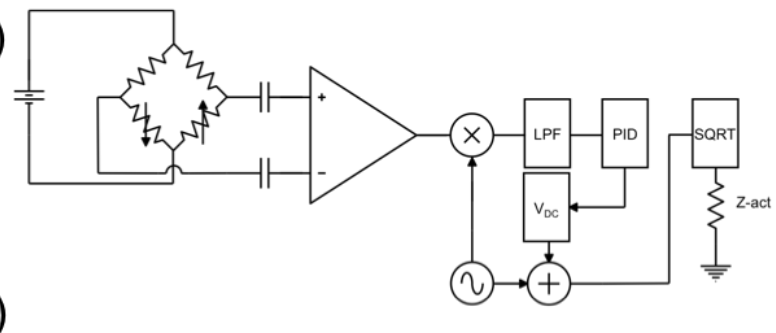

b)
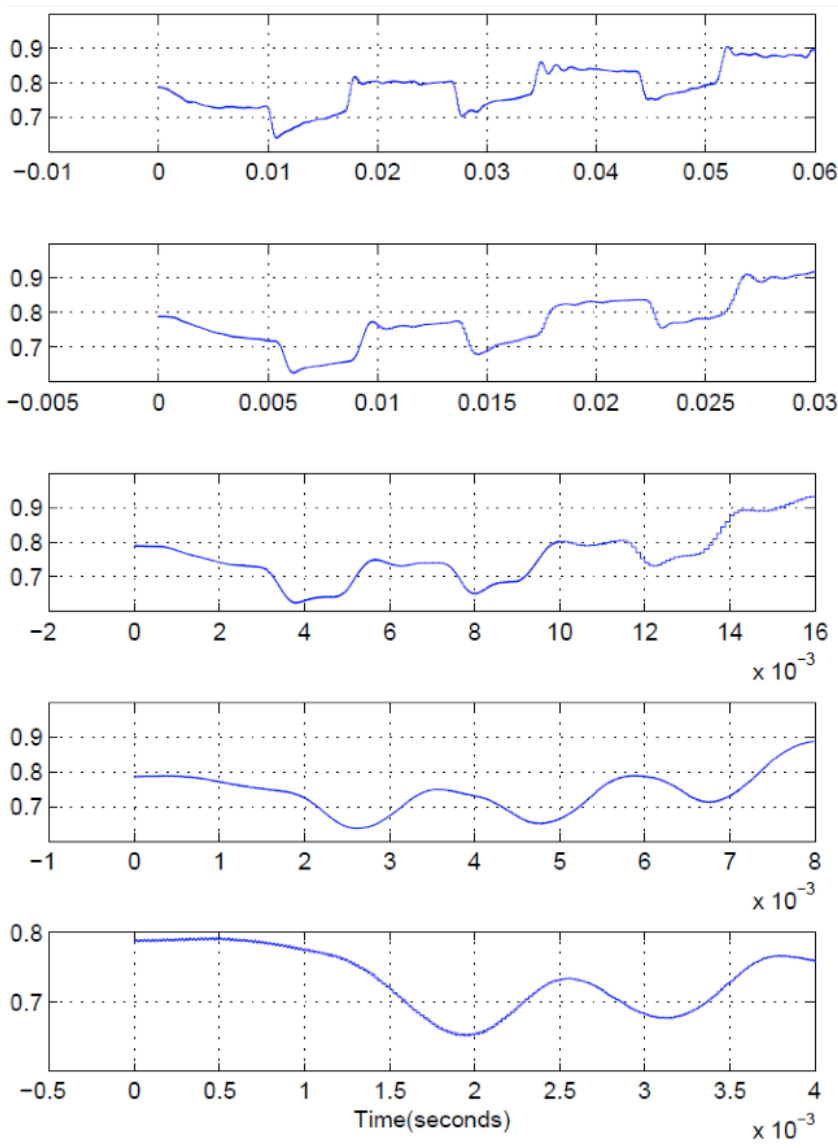

Figure 7a) Block diagram of the intermittent contact AFM used to perform b) line scans at progressively higher speeds.
The sc-AFM can also operate in contact mode, where the cantilever is brought into contact with the sample. A DC offset is experienced between the sense and dummy piezoresistors on the sc-AFM when contact is made with the sample. As the tip is scanned across the surface, static deflections change the output of the piezoresistive bridge signal, and a PID may be used to adjust the output voltage of the Z-actuator to maintain a constant height above the sample, as shown in Fig 8a. Fig 8b shows the line scan of a $100 \mathrm{~nm}$ calibration grating obtained with a progressively higher scan rate. Box-car averaging is used to reduce the noise in the data. Unlike the device used in the intermittent contact, this device contains optimized heater placements in the chevron lateral actuator, allowing for higher frequency of operation. The faster scan rates do not limit the scan range.

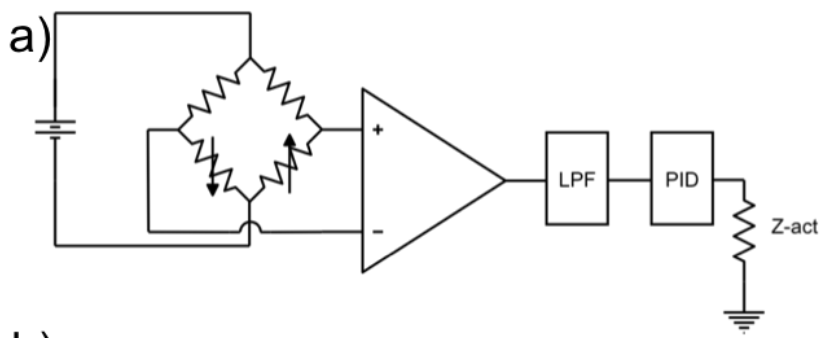

b)
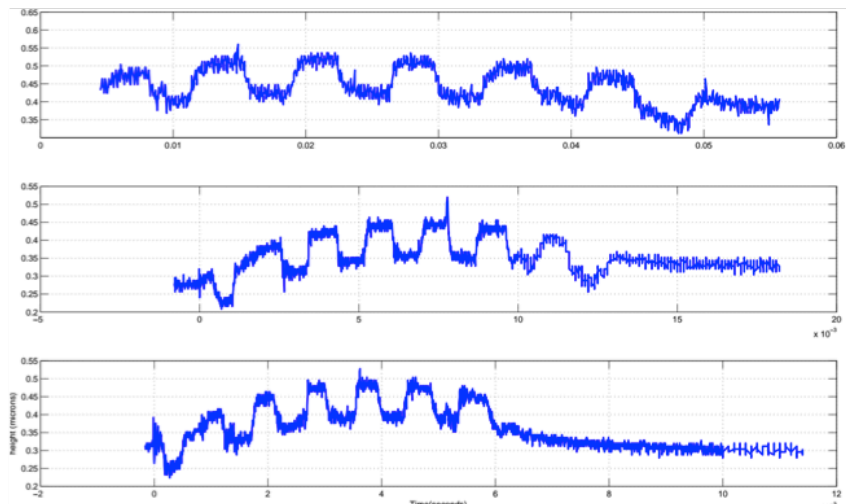

Figure 8a) Block diagram of DC coupled contact AFM used to perform b) line scans at progressively higher speeds (50ms, $15 \mathrm{~ms}$ and $6 \mathrm{~ms}$ ).

Finally the sc-AFM is operated in open-loop while applying an AC signal directly to the piezoresistive bridge, as depicted in Fig. 9a. After the approach, the device scans across the surface with the tip in contact. Fig. $9 \mathrm{~b}$ shows the line scan of a $100 \mathrm{~nm}$ tall DVD sample. Irregular bumps are seen in the line scan, as the DVD contains significant variations in the distribution of peaks and valleys. As the line is scanned at a progressively faster rate, the quality of data does not diminish. Rates beyond $200 \mathrm{~Hz}(400$ lines/sec) are possible using this method, suggesting that video rate AFM is achievable with the sc-AFM system. 


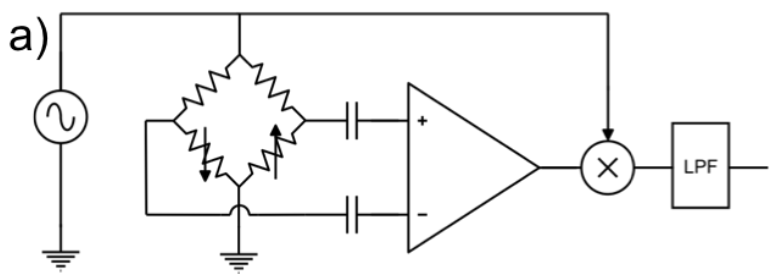

b)

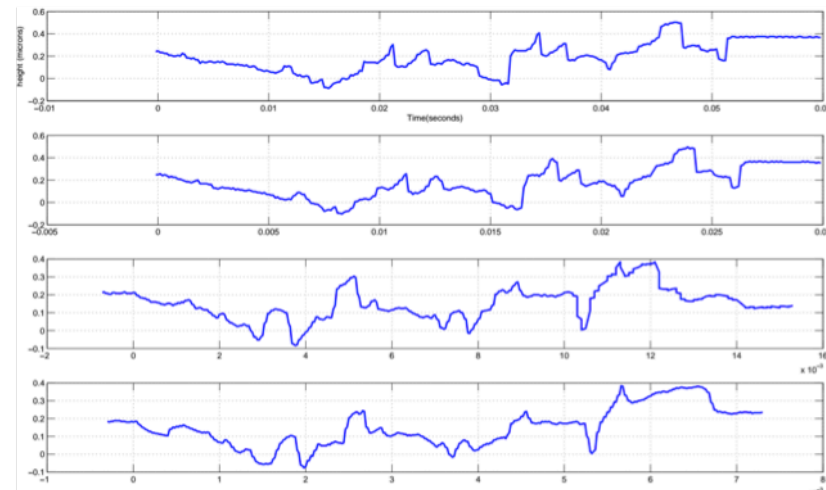

Figure 9a) Block diagram of AC-coupled contact AFM used to perform b) line scans at progressively higher speeds (60ms, 30ms, $15 \mathrm{~ms}, 7 \mathrm{~ms}$ ).

\section{LONG-TERM RELIABILITY}

In [6], images of a 22nm SRAM bank were reported using Scanning Thermal Microscopy (SThM). The topography was not visible by AFM, and SThM images were severely distorted. The present device obtained images of the same sample, clearly revealing the contact-level structure of the 6-transistor bit cell as seen in Fig. 10a. The tip wear was characterized by imaging the same area over the period of several days. Ghosting was observed in the first 100 images, presumably due to residual polymer coverage of the tip from the fabrication process used to release the devices. Imaging operation removes the polymer and sharpens the image. No observable changes occurred after 100 cycles and lower resolution images were taken after 700 cycles (Fig. 10b-d), in order to accelerate the distance traversed by the tip. After $9000+$ cycles, no additional tip wear was observed.
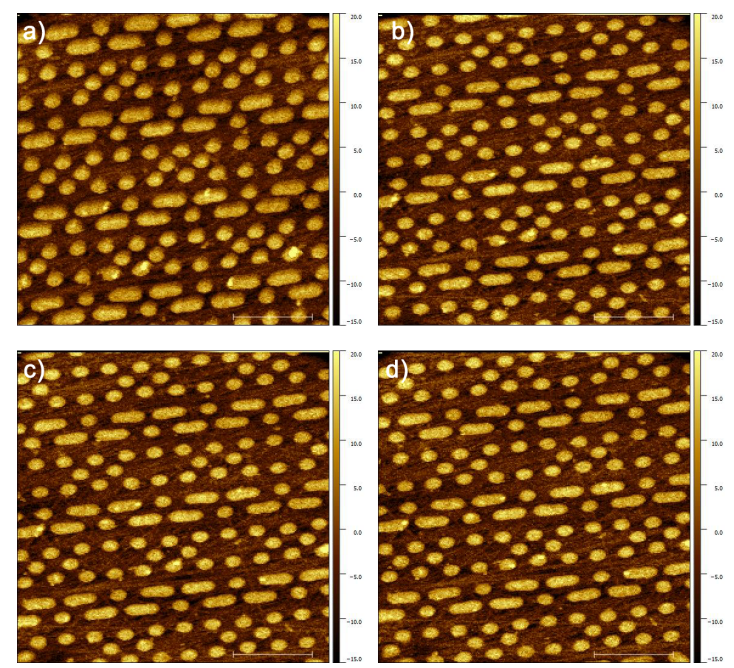

Figure 10: Imagine of $22 \mathrm{~nm}$ SRAM cells after a) 1 cycle, b) 250 cycles, c) 500 cycles, d) 700 cycles
Conventional cantilevers are typically replaced after 50 images under the reported operating conditions. The $<1 \mu \mathrm{m}$ of lateral drift occurs due to temperature changes in the room over the course of the imaging experiment.

\section{CONCLUSION}

The sc-AFM has been used to demonstrate consistent imaging over a $15 \times 15 \mu \mathrm{m}$ area with a vertical resolution of $1.5 \mathrm{~nm}$, both $200 \%$ better than previously reported. Quasi-static operation has been investigated, where characteristics such as adhesion can be probed. High speed line scanning has been performed, showing that up to 400 lines of data can be obtained in one second. Tip wear has been characterized through a series of lifetime tests. Scanning over the same sample area does not degrade the tip, even over the course of 9000 scans.

Near-term goals are to make video rate sc-AFM a reality and to provide high quality, fast and accurate imaging operation with minimal tip wear.

\section{REFERENCES}

[1] "CMOS-MEMS atomic force microscope," Transducers '11, N. Sarkar and R. Mansour (2011)

[2]"High-speed, large scale imaging with the atomic force microscope," JVST B, R.C. Barret and C. Quate (1991) [3]"A high-speed atomic force microscope for studying biological macromolecules," T. Ando et. al., Proc. Natl. Acad. Sci. (2001)

[4]"Design and control of multi-actuated AFM for large-range and high-speed imaging," Bozchalooi et. al., Ultramcrscpy, (2016)

[5]"A distortion-free single-chip atomic force microscope with 2DOF isothermal scanning," D. Strathearn et. al., Transducers '15.

[6]"A single-chip SPM for topographical and thermal metrology at the nanometer scale," N. Sarkar et. al., MEMS 2016.

\section{CONTACT}

*G.Lee, tel: +1-519-888-4567; g4lee@uwaterloo.ca 